\title{
Editorial
}

\section{Immunotherapy in Gastrointestinal Cancer: Where Do We Stand?}

\author{
Andrea Tannapfel ${ }^{a} \quad$ Anke Reinacher-Schick ${ }^{b}$ \\ ${ }^{a}$ Institute of Pathology, Ruhr-University Bochum, Bochum, Germany; ${ }^{b}$ Department of Hematology, Oncology and \\ Palliative Care, Ruhr-University Bochum, Bochum, Germany
}

Gastrointestinal (GI) malignancies are the most common cancers worldwide and have a huge impact on cancerassociated mortality. For many decades, patients with GI cancers were treated with surgery, radiotherapy or chemotherapy alone or in combination. Despite these therapeutic measures, prognosis of patients with advanced or metastatic disease was still dismal. Only patients diagnosed at an early stage had favorable outcomes. With the introduction of targeted agents into GI oncology more than 10 years ago prognosis improved significantly for a subset of patients. Examples were the addition of trastuzumab to combination chemotherapy in HER2-positive advanced gastric cancer [1] or anti-EGFR agents in RAS wild-type metastatic colorectal cancer [2]. However, improvement in overall survival is mostly small, sometimes summing up to only a few months. In addition, there were few major breakthrough reports, i.e., the discovery of c-KIT alterations in GI stromal tumors [3] and the impressive activity of tyrosine kinase inhibitors, such as imatinib, in this disease. But GI stromal tumors are very rare, and, therefore, only a smaller number of all patients with GI cancers have benefitted from this discovery.

Therefore, novel strategies to fight cancer were badly needed. Presently, it seems that immunotherapy may be the answer. In the past, mostly cytokines, such as interferon alpha or interleukin-2, were approved for some cancers (renal cell carcinoma and melanoma) rendering moderate activity. Also, cancer vaccines that have been developed so far have not shown significant response rates.

\section{Checkpoint Inhibition as a New Concept to Target Cancer}

The immune system is maintained by a diligent interplay of inhibitory and stimulating signals. In the 1990s, the CTLA-4 receptor was discovered, and in 1996, it was demonstrated for the first time in an experimental model that inhibition of the CTLA-4 receptor enhanced antitumor activity and mice were cured of their disease [4]. In parallel, the PD-1/PD-L1 was discovered as another important immune checkpoint system characterizing the function of receptors and ligands [5]. In the following years, a large number of preclinical and clinical studies followed, and in 2011, the FDA approved the CTLA-4 antagonist ipilimumab for the treatment of advanced malignant melanoma. In the past years, we experienced approval of several PD-1 and PD-L1 antagonists for a large variety of cancers leading to the establishment of immunooncology as a completely new strategy to fight cancer. While the classic cancer therapies, such as chemotherapy, radiation, and also most targeted agents, attack the cancer cell itself, checkpoint inhibitors are directed against stromal components, i.e., the immune cells. They activate these tumor-infiltrating and tumor-surrounding immune cells, restore immune response against the cancer, and thereby achieve objective responses which are especially durable. Subsequently, the Nobel Prize for Medicine in 2018 was awarded to James Allison and Tasuku Honjo for their discovery of the checkpoint system, expressing the high importance of their experimental work towards this recent revolution in cancer therapy.

\section{Immunooncology in GI Cancers - Is It a New Standard Yet?}

In spite of the major achievements in some solid tumors, such as malignant melanoma or non-small cell lung cancer, checkpoint inhibition has not shown similar success in GI malignancies so far. In particular, there were some negative studies in gastric or pancreatic cancer. However, some significant steps have been made, mostly

\section{KARGER}

() 2019 S. Karger AG, Basel
Prof. Dr. Anke Reinacher-Schick Department of Hematology, Oncology and Palliative Care Ruhr-University Bochum, Gudrunstraße 56 DE-44791 Bochum (Germany)

E-Mail Anke.Reinacher@ rub.de 
in small subsets of cancers, such as those with microsatellite instability-high (MSI-H) genotype. This molecular feature is characteristic for a number of GI cancers, such as some hereditary (Lynch) or sporadic colorectal cancers, gastric cancers, as well as cancers of the small intestine and biliary tract. In fact, in 2017, the FDA approved pembrolizumab as the first tissue/site agnostic treatment for adult or even pediatric patients with MSI-H or defective mismatch repair system progressing after prior treatment and lacking alternative treatment options. Moreover, patients with colorectal cancer having progressed after 5-FU, oxaliplatin, and irinotecan may also receive pembrolizumab. The biological mechanism which makes MSI-H cancers so sensitive to checkpoint inhibition lies in their high number of mutations which lead to the production of neoantigens, which in turn causes extensive immune cell infiltration. Pathologists have long been aware of the dense immune cell infiltrates in MSI-H cancers, in particular colorectal cancer of Lynch origin.

The present developments in immunooncology seem to be just the beginning of this new era in cancer treatment. In fact, not all patients respond to checkpoint inhibition, and mechanisms for treatment resistance as well as biomarkers for treatment response are being explored by various research groups.

\section{Biomarkers for Treatment Response to Checkpoint Inhibition}

Several biomarkers for immunotherapy are being presently analyzed, including tumor-infiltrating immune cells, immune-regulating factors, as well as host genomic factors. In contrast to other biomarkers (e.g., RAS), reliable immunotherapy biomarkers are limited because of our incomplete understanding of how the immune system of a given tumor works and how given immunotherapies modify the tumor microenvironment. Some of the markers of investigation are both prognostic and also predictive mark- ers, even more complicating the search. The evaluation and determination of cutoffs are far from being standardized. Presently, most groups accept PD-L1 as well as MSI$\mathrm{H}$ as a sensible biomarker for checkpoint inhibitory agents.

In this issue of Visceral Medicine, various aspects of immunotherapy in GI cancers are reviewed. First, immunologic biomarkers and biomarkers for immunotherapy are described by Martin and Märkl et al. [6] reviewing the role of PD-L1, immune-infiltrating lymphocytes, and tumor mutational burden in immunooncology for GI cancers. Furthermore, Canbay and colleagues [7] report the present knowledge of immunotherapy in liver cancer related to hepatitis and related to alcoholic or nonalcoholic steatohepatitis. Especially with 2 positive phase III trials, checkpoint inhibitors were approved by the FDA for second-line treatment in hepatocellular carcinoma. Interestingly, some studies also explore the combination of locoregional treatment and immunooncology with promising results. In addition, Ettrich and colleagues [8] review the present knowledge of immunotherapy in pancreatic and biliary cancer where most substances are less effective. Here, the role of the stroma components as intrinsic mechanisms of resistance and modes to transform "cold" cancers into "hot" lesions are also reported. The authors also extensively describe present vaccination strategies in pancreatic and biliary tract cancer which, however, are still at the beginning. Additionally, Stein et al. [9] review the role of checkpoint inhibition in gastric cancer where the substances seem effective especially in MSI-H cancers, less in $\mathrm{PD}-\mathrm{L} 1$ positive cancers. Finally, a number of case reports on immunotherapy in GI cancers complete this issue of Visceral Medicine.

\section{Disclosure Statement}

Honoria: Amgen, Roche, Pfizer, Sanofi-Aventis, Merck Serono, Shire, Celgene, Lilly, BMS, Servier, Baxalta, MSD. Advisory board member: Amgen, Roche, Pfizer, Sanofi-Aventis, Merck Serono, Celgene, Lilly, BMS, Servier, Baxalta, MSD. Studies sponsored by: Roche, Sanofi-Aventis, Celgene, Ipsen.

\section{References}

1 Bang YJ, Van Cutsem E, Feyereislova A, Chung HC, Shen L, Sawaki A, et al.; ToGA Trial Investigators. Trastuzumab in combination with chemotherapy versus chemotherapy alone for treatment of HER2-positive advanced gastric or gastro-oesophageal junction cancer (ToGA): a phase 3, open-label, randomised controlled trial. Lancet. 2010 Aug; 376(9742):687-97.

2 Van Cutsem E, Köhne CH, Hitre E, Zaluski J, Chang Chien CR, Makhson A, et al. Cetuximab and chemotherapy as initial treatment for metastatic colorectal cancer. N Engl J Med. 2009 Apr;360(14):1408-17.
3 Hirota S, Isozaki K, Moriyama Y, Hashimoto K, Nishida T, Ishiguro S, et al. Gain-of-function mutations of c-kit in human gastrointestinal stromal tumors. Science. 1998 Jan; 279(5350):577-80

4 Leach DR, Krummel MF, Allison JP. Enhancement of antitumor immunity by CTLA4 blockade. Science. 1996 Mar;271(5256): 1734-6.

5 Freeman GJ, Long AJ, Iwai Y, Bourgue K, Chernova T, Nishimura $\mathrm{H}$, et al. Engagement of the PD-1 immunoinhibitory receptor by a novel B7 family member leads to negative regulation of lymphocyte activation. J Exp Med. 2000 Oct;192(7):1027-34.
6 Martin B, Märkl B. Immunologic biomarkers and biomarkers for immunotherapies in gastrointestinal cancer. Visc Med. 2019. https:// doi.org/10.1159/000496565.

7 Gottlieb A, Best J, Canbay A. Implications of Immunotherapy in hepatobiliary tumors. Visc Med. 2019. https://doi.org/10.1159/000496755.

8 Perkhofer L, Beutel AK, Ettrich TJ. Immunotherapy: pancreatic cancer and extrahepatic biliary tract cancer. Visc Med. 2019. https:// doi.org/10.1159/000497291.

9 Stein A, Thuss-Patience PC. Immunotherapy in esophagogastric adenocarcinoma. Visc Med. 2019. https://doi.org/10.1159/000497292. 